\title{
Effect of Administration Duration of Low Dose Methotrexate on Development of Acute Kidney Injury in Rats
}

\author{
Xiao $\mathrm{Li}^{1}$, Erika Abe ${ }^{1}$, Yukako Yamakawa ${ }^{1,2}$, Go Yoneda ${ }^{1}$, Rika Fujino ${ }^{2}$, Mami Yamashita ${ }^{1}$, Yuumi lida ${ }^{1}$, Hirofumi Jono ${ }^{1,2}$ and Hideyuki Saito ${ }^{1,2^{*}}$ \\ ${ }^{1}$ Department of Clinical Pharmaceutical Sciences, Graduate School of Pharmaceutical Sciences, Kumamoto University, 5-1 Oe-honmachi, Chuo-ku, Kumamoto, Japan
}

${ }^{2}$ Department of Pharmacy, Kumamoto University Hospital, 1-1-1 Honjo, Chuo-ku, Kumamoto, Japan

"Corresponding author: Hideyuki Saito, Department of Pharmacy, Kumamoto University Hospital, 1-1-1 Honjo, Chuo-ku, Kumamoto 860-8556, Japan, Tel: +81-96-373-5820; E-mail: saitohide@fc.kuh.kumamoto-u.ac.jp

Received date: June 20, 2016; Accepted date: July 13, 2016; Published date: July 20, 2016

Copyright: $\odot 2016 \mathrm{Li} \mathrm{X}$, et al. This is an open-access article distributed under the terms of the Creative Commons Attribution License, which permits unrestricted use, distribution, and reproduction in any medium, provided the original author and source are credited.

\begin{abstract}
Methotrexate (MTX) is currently utilized as a key drug in treatment of both malignant tumors and rheumatoid arthritis. However, MTX treatment is often associated with various side effects, such as pulmonary damage, hepatotoxicity and nephrotoxicity. Recent report also revealed that, even though total administered dosage of MTX was same, longer duration of MTX administration caused more severe adverse effect rather than short duration of its administration in clinical. Despite the importance of appropriate usage of MTX, the mechanism of kidney injury caused by the difference in duration of MTX administration remains still unknown. Here, we established animal models to determine the effect of administration duration on MTX-induced kidney injury and evaluated the significant factor and mechanism responsible for MTX caused-kidney injury. The dosage of MTX (25 mg/kg) were intraperitoneally injected by short-administration $(25 \mathrm{mg} / \mathrm{kg}$ by 1 injection: Short-MTX) or long-administration ( 5 $\mathrm{mg} / \mathrm{kg}$ by 5 injections: Long-MTX), respectively. In Long-MTX group, body weight, water intake, and urine volume were significantly decreased. Urea nitrogen and creatinine (CRE) in urine were obviously decreased in Long-MTX group, while blood urea nitrogen (BUN) and CRE in serum were increased in Long-MTX group. In addition, LongMTX group showed the significant increase of both neutrophil gelatinase-associated lipocalin ( $\mathrm{N}$-gal) and kidney injury molecule 1 (Kim-1), kidney injury markers. Interestingly, renal MTX concentrations in Long-MTX group was higher than those in Short-MTX group. Moreover, 4-hydroxynonenal (4-HNE) and malondialdehyde (MDA), reliable oxidative stress markers, were significantly increased in Long-MTX group. Taken together, the present findings suggest that longer duration of MTX administration caused a higher MTX accumulation in kidney, thereby leading to kidney injury through an increase in oxidative stress.
\end{abstract}

Keywords: Methotrexate; Administration duration; Kidney injury; Oxidative stress

\section{Abbreviations}

AKI: Acute Kidney Injury; BUN: Blood Urea Nitrogen; CRE: Creatinine; 4-HNE: 4-Hydroxynonenal; KIM-1: Kidney Injury Molecule-1; MDA: Malondialdehyde; MTX: Methotrexate; N-GAL: Neutrophil Gelatinase-Associated Lipocalin

\section{Introduction}

Methotrexate (MTX), an antifolate, is a drug widely used in the treatment of patients with various diseases, such as, malignant tumors [1], rheumatoid arthritis (RA) [1,2], and ectopic pregnancy [3]. The clinical application of MTX has been increased since 1980s [4], and MTX is currently the most frequently disease-modifying drug both in adult RA and juvenile idiopathic arthritis $[5,6]$. Moreover, it is also widely used in malignant tumors, such as leukemia, lymphoma, choriocarcinoma, head and neck cancer, and osteogenic sarcoma [7].

Although MTX is currently utilized as a key drug in the treatment of both malignant tumors and RA, the efficacy of MTX is often limited by severe side-effects [8]. It is well-documented that MTX exhibits adverse effects including myelosuppression, mucositis, and kidney damage [9]. Of those side effects, kidney injury is one of the most frequent complications of MTX treatment. Because MTX is largely excreted into urine via kidney in its original form, MTX-induced renal dysfunction results in sustained and elevated plasma MTX concentrations, which in turn may lead to cause enhancement of other MTX toxicities [10]. Despite the importance of appropriate usage of MTX, the mechanism underlying MTX-induced kidney injury remains still unknown. In addition, previous studies have also shown that neither dosage nor plasma concentration may be a suitable index to predict MTX-induced kidney injury [11-13].

MTX has been used in a wide range of doses in clinical treatments from low dose of $20 \mathrm{mg} / \mathrm{m}^{2}$ to high dose of $33,000 \mathrm{mg} / \mathrm{m}^{2}$ (1,000 times higher than the low dose) [14]. It has been reported that high dose MTX could cause kidney injury by the precipitation of MTX [12]. Because the solubility of MTX in urine is directly influenced by $\mathrm{pH}$, MTX is crystallized and precipitated in acid urine [12]. On the other hand, low dose MTX-induced kidney injury also have been frequently observed in clinical practice [13], which is unexplained by crystalinduced injury mechanism. Moreover, it has shown that the toxicity of MTX is associated with the infusion duration in clinical usage [15]. Recent studies also indicated that longer duration of MTX administration caused more severe adverse effect rather than short duration of its administration in patients [16]. However, the mechanism of kidney injury caused by the difference in duration of MTX administration has yet to be determined, and a suitable model to explore its mechanism is not yet available. 
The purpose of this study was to establish a suitable animal model to study the mechanism involved in MTX-induced kidney injury and the effect of administration duration.

\section{Materials and Methods}

\section{Drugs and chemicals}

MTX was obtained from Pfizer Inc. (New York, USA). MTX standard chemical was purchased from Wako Pure Chemical Industries, Ltd. (Osaka, Japan). N-gal antibody was purchased from R\&D Systems (Minneapolis, USA), 4-HNE antibody was purchased from Abcam plc. (Cambridge, UK). All other chemicals used in this study were of the highest purity grade available.

\section{Animal experiments}

Male Wistar rats, each 10 weeks old (300 g), were obtained from Kyudo, Co, Ltd (Tosu, Japan). MTX administration method was based on clinical usage $[17,18]$. MTX $(25 \mathrm{mg} / \mathrm{kg})$ were intraperitoneally injected by short- or long-administration duration. Short administration duration control group (saline, $\mathrm{n}=6$ : Short-Con) and MTX group (25 mg/kg MTX saline solution, $n=6$ : Short-M) were administered by 1 injection at day 1 . Long administration duration control group (saline, $\mathrm{n}=6$ : Long-Con) and MTX group (5 mg/kg MTX solution, $\mathrm{n}=6$ : Long-MTX) were administered by 5 injections from day 1 to day 5, once a day. Analysis of biochemistry, such as urea nitrogen and creatinine (CRE), was performed by SRL Inc. (Tokyo, Japan). All procedures for animal experiments were approved by Kumamoto University ethical committee concerning animal experiments (B25-120R1), and animals were treated in accordance with the Guidelines of Kumamoto University for the care and use of laboratory animals.

\section{Sample collection}

Serum samples were collected on day 7 and centrifuged (3,000 g, 10 $\mathrm{min})$. Urine samples were collected by metabolism cages every $24 \mathrm{hrs}$ from 1 day before administration. Kidney tissue samples were collected on day 7. All samples were stored at $-80^{\circ} \mathrm{C}$ until analysis.

\section{Western blot analysis}

The homogenized kidney sample was denatured with SDS buffer, separated by $10 \%$ Tris-Tricine SDS-PAGE, and transferred onto nitrocellulose membrane. The membrane was incubated with $\mathrm{N}$-gal antibody (R\&D Systems) for overnight at $4^{\circ} \mathrm{C}$, and then incubated with anti-goat IgG antibody (Santa Cruz Biotechnology, Inc., Texas, USA) for $1 \mathrm{hr}$ at room temperature. The membrane was incubated with detection reagent (ECL Advance Western Blotting Detection Kit; GE Healthcare UK Ltd, Buckinghamshire, England), and detected with LAS4000 (Fujifilm) and analyzed with Image J.

\section{Enzyme-Linked Immuno-Sorbent Assay (ELISA)}

The kidney tissue $(500 \mathrm{mg}$ ) was homogenized in $1 \mathrm{ml}$ of PBS. ELISA for Kim-1 was performed by using Kim-1 ELISA kit (R\&D Systems), according to the manufacturer's protocol.

\section{Liquid chromatography/mass spectrometry/MS (LC/MS/MS) analysis}

MTX concentration was measured by using API $3200^{\mathrm{TM}}$ LC/MS/MS system (AB SCIEX, Foster City, CA, USA) with triple quadruple mass spectrometer following positive ion mode: MTX, $\mathrm{m} / \mathrm{z}$ 455.021; MTX d3, m/z 308.200. Source parameters were optimized to get highest analyte peak. The chromatographic separation was performed on $2.1 \times 50 \mathrm{C} 18$ column $(3 \mu \mathrm{m}$ particle size). The mobile phase consisting ammonium acetate buffer $(1 \mathrm{nM}$ ammonium acetate and $0.2 \%$ formic acid $\mathrm{v} / \mathrm{v})$ and acetonitrile $(82: 18 \mathrm{v} / \mathrm{v})$.

\section{Determination of MTX in kidney tissue}

The kidney tissue $(500 \mathrm{mg})$ was homogenized, centrifuged $(15,000$ $\mathrm{g}, 3 \mathrm{~min}$ ), and the supernatant was collected. The perchloric acid (25 $\mu \mathrm{l})$ was added into $100 \mu \mathrm{l}$ supernatant. The samples were centrifuged, and the supernatant $(10 \mu \mathrm{l})$ was injected into LC/MS/MS system.

\section{Determination of MTX in urine}

The Evolute ${ }^{\mathrm{TM}} \mathrm{ABN}$ cartridge was activated with $1 \mathrm{ml}$ of $\mathrm{MeOH}$ and $1 \mathrm{ml}$ of $0.1 \%$ aqueous formic acid $\mathrm{v} / \mathrm{v}, 0.1 \mathrm{ml}$ of centrifuged $(15,000 \mathrm{~g}$, $10 \mathrm{~min}$ ) and diluted (1:1 with $1 \%$ aqueous formic acid $\mathrm{v} / \mathrm{v})$ urine sample were loaded into the cartridge. The cartridge was the washed with aqueous $5 \% \mathrm{MeOH}$ solution. Finally, the analyses were eluted with $300 \mu \mathrm{l}$ of $\mathrm{MeOH}$. The samples were evaporated the equate under vacuum at $45^{\circ} \mathrm{C}$ and re-dissolved in mobile phase. The samples $(10 \mu \mathrm{l})$ was then injected into LC/MS/MS system.

\section{Histological analysis}

Paraffin-embedded $4 \mu \mathrm{m}$-thick sections were used for HematoxylinEosin ( $\mathrm{H} \& \mathrm{E})$ staining and immunohistochemical analysis. For $\mathrm{H} \& \mathrm{E}$ staining, the slides were stained with hematoxylin for $15 \mathrm{~min}$, and stained with eosin for 2 min.

\section{Immunohistochemistry}

Paraffin-embedded $4 \mu$ m-thick sections were used for immunohistochemistry. Deparaffinized sections were activated antigen with proteinase $\mathrm{K}$ (Dako). The slides were incubated with 4 -HNE antibody (200 times dilution) overnight at $4^{\circ} \mathrm{C}$, and then incubated with secondary anti-mouse IgG antibody (GE Healthcare UK Ltd., 200 times dilution) at room temperature for $1 \mathrm{hr}$. Reactivity was visualized with the DAB Liquid System (Dako Denmark A/S, Glostrup, Denmark).

\section{Malondialdehyde (MDA) analysis}

The kidney tissue $(50 \mathrm{mg}$ ) was collected and homogenized in $250 \mu \mathrm{l}$ RIPA Buffer. MDA analysis was performed by using MDA assay kit (Cayman Chemical., Michigan, USA), according to the manufacturer's protocol.

\section{Statistical analysis}

All data was expressed as mean \pm SD. Difference between groups were tested for statistical significance using Student's t-test. P-values of $<0.05$ were considered statistically significant. 
Citation: Li X, Abe E, Yamakawa Y, Yoneda G, Fujino R, et al. (2016) Effect of Administration Duration of Low Dose Methotrexate on

Page 3 of 6

\section{Results}

\section{MTX-induced kidney injury by short or long administration duration}

We first sought to establish the animal model exhibiting kidney injury caused by MTX to determine whether the administration duration affects the MTX-induced kidney injury. The dosage of MTX $(25 \mathrm{mg} / \mathrm{kg})$ were intraperitoneally injected by Short-administration $(25$ $\mathrm{mg} / \mathrm{kg}$ by 1 injection) or Long-administration $(5 \mathrm{mg} / \mathrm{kg}$ by 5 injections), respectively. As shown in Figure 1a, body weight was significantly decreased in long administration duration of MTX (LongMTX) group compared to short administration duration MTX (ShortMTX) group.

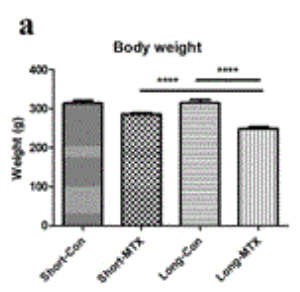

b

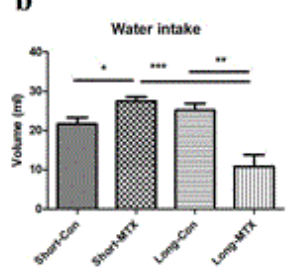

d

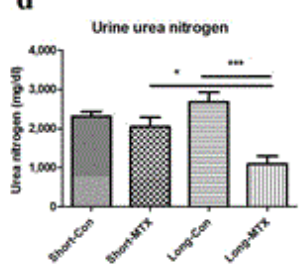

f

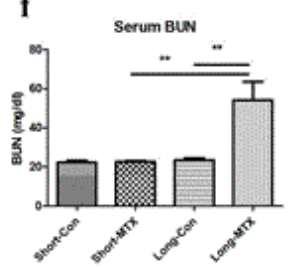

c

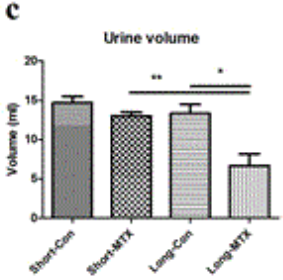

e
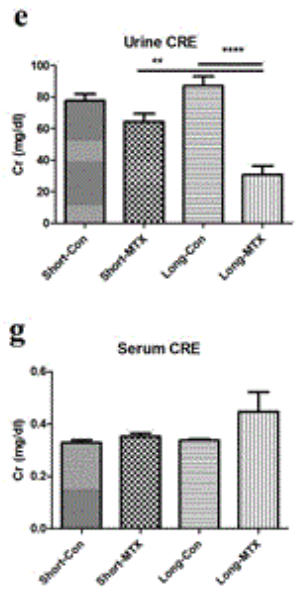

Figure 1: MTX-induced kidney injury by short or long administration duration. Body weight (a), water intake (b), and urine volume (c) were evaluated in the MTX-administrated rats by short-duration (Short-MTX, $\mathrm{n}=3$ ) or long-duration (Long-MTX, $\mathrm{n}=6$ ). Urine urea nitrogen $(\mathrm{d})$, urine CRE (e) and serum BUN (f), serum CRE (g), were also determined in the MTX-administrated rats. Each bar represents the mean $\pm \mathrm{SD} .{ }^{*}: \mathrm{p}<0.05 ;^{* *}: \mathrm{p}<0.01{ }^{* * *}:$ $\mathrm{p}<0.001 ;{ }^{* * *}: \mathrm{p}<0.0001$.

In Long-MTX group, water intake and urine volume were also decreased markedly (Figures $1 \mathrm{~b}$ and 1c), suggesting MTX-induced kidney injury. To further evaluate MTX-induced kidney injury, urea

$$
\text { MTX) group. }
$$

nitrogen and CRE were also measured in urine and serum samples. In urine, urea nitrogen and CRE were obviously decreased in Long-MTX group (Figures $1 \mathrm{~d}$ and 1e). In contrast, serum BUN and CRE were increased in Long-MTX group (Figures $1 \mathrm{f}$ and $1 \mathrm{~g}$ ). Interestingly, no significant changes were observed in Short-MTX group (Figures $1 \mathrm{~d}-1 \mathrm{~g})$. Those results suggested that longer duration of MTX administration caused more severe kidney injury rather than short duration of its administration in rat model.

\title{
Kidney tissue injury caused by long-MTX administration
}

By using established rat model, we attempted to determine the kidney tissue injury caused by long-MTX administration. As shown in Figure 2a, histological analysis showed that Long-MTX administration caused tubular and glomerular injury in cortex. Kidney hyperemia, tubular damage, and glomerulus atrophy were also observed in kidney tissue of Long-MTX group.

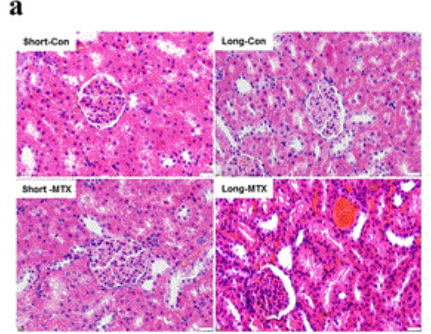

c

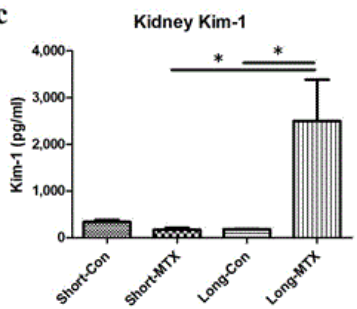

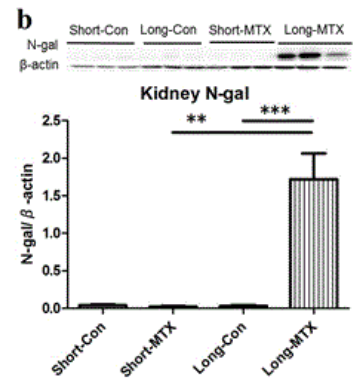

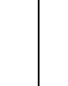

.


Page 4 of 6

accumulation in kidney and built a simple method to evaluate MTX concentration in renal tissue (Figure 3a). The calibration curve was established with homogenized blank kidney sample and standard MTX chemical at the concentration between $15.625 \mathrm{ng} / \mathrm{ml}$ to 500 $\mathrm{ng} / \mathrm{ml}$ (Figure 3b). As shown in Figure 3c, renal MTX concentrations in Long-MTX group was higher than those in Short-MTX group. As expected, in Long-MTX group, total amount of excrete MTX in urine was decreased (Figure 3c), suggesting that long-MTX administrationinduced severe kidney injury was caused by the accumulation of MTX in renal tissue.
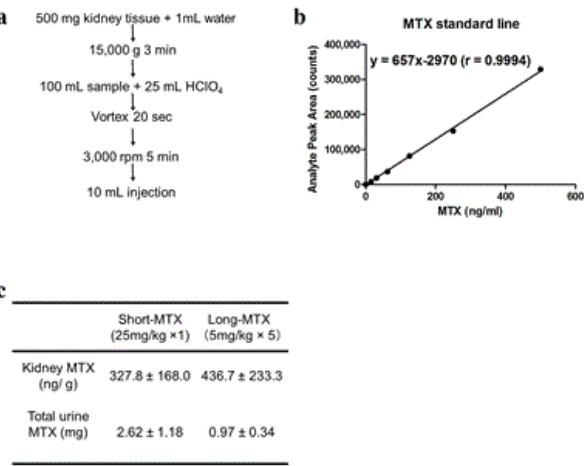

Figure 3: MTX concentration in and kidney and total MTX in urine. (a) Sample preparation method for measuring MTX concentration in kidney. (b) Standard line of renal MTX determination by LC-MS/MS. (c) Renal MTX concentration and total urine MTX in MTX-administrated rats.

\section{Involvement of oxidative stress in kidney injury caused by Long-MTX administration}

To further explore the mechanism of kidney injury caused by MTX accumulation in kidney, we evaluated 4-HNE, major end products of lipid peroxidation and known as an oxidative stress marker [19]. Immunohistochemical analysis showed that 4-HNE was increased in kidney tissue of Long-MTX group (Figure 4a). a
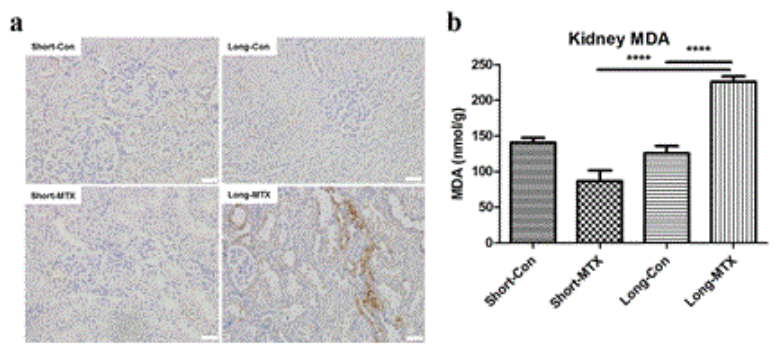

Figure 4: Involvement of oxidative stress in MTX-induced kidney injury. Immunohistochemical analysis for 4-HNE (a) and MDA analysis (b) in the kidney of MTX-administrated rats. Scale bars represent $20 \mu \mathrm{m} .{ }^{* * *}: \mathrm{p}<0.0001$.
Moreover, MDA, an another oxidative stress marker [20], was also significantly increased in the kidney tissue of Long-MTX group (Figure 4b), suggesting that the MTX accumulation in kidney by LongMTX administration caused kidney injury through an increase in oxidative stress.

\section{Discussion}

In clinical practice, MTX is considered as a key drug in the treatment of both malignant tumors and RA. However, MTX treatment is often associated with various toxicities, such as kidney injury, which unexpectedly result in the interruption or discontinuation of medical treatment. Moreover, although it has been reported that the toxicity of MTX is associated with the infusion duration $[15,16]$, the mechanism of kidney injury caused by the difference of MTX administration duration has yet to be determined. In the present study, we successfully established the animal model reflecting the clinical MTX-induced kidney injury caused by longer duration of MTX administration, and found that MTX accumulation in renal tissue by Long-MTX administration causes kidney injury through an increase in oxidative stress.

One of the interesting finding in this study is that, even though the administrated dosage of MTX was same $(25 \mathrm{mg} / \mathrm{kg})$, Long-MTX administration caused more severe kidney injury than Short-MTX administration. Goldie et al., reported the duration of MTX administration was important factor in MTX-induced toxicity [15]. Recent report also showed that shorter duration of MTX administration was more beneficial in view of reducing toxicity and enhancing central nervous system pharmacokinetics for lymphomas patients. In our experiments, the dosage of MTX $(25 \mathrm{mg} / \mathrm{kg})$ were intraperitoneally injected to the rats by Short-administration (25 $\mathrm{mg} / \mathrm{kg}$ by 1 injection) or Long-administration $(5 \mathrm{mg} / \mathrm{kg}$ by 5 injections), respectively. While the same dosage of MTX was administrated, longer duration of MTX administration caused more severe kidney injury than short duration of its administration in rat model (Figure 1). It is well-documented that neither dosage nor plasma concentration may be suitable index to predict MTX-induced kidney injury [11-13]. Previous study showed that, in high dose of MTX, toxicity was not related to plasma MTX area under the curve [21]. Meanwhile, MTX rapidly eliminated from the plasma at low dose, and plasma MTX concentration was also unrelated to toxic response, indicating MTX plasma levels may not be a reliable and not appropriate index for therapeutic drug monitoring [11]. As expected, no obvious correlation between serum MTX concentration and kidney tissue injury was observed (Data not shown). Interestingly, our further analysis focusing on MTX concentration in kidney tissue, suggested that MTX accumulation in kidney by Long-MTX administration. Since previous studies have shown that oxidative stress plays important roles in MTX-induced kidney injury [22], we also determined the oxidative markers, $4 \mathrm{HNE}$ and MDA, in kidney. The result shows longer administration duration of MTX caused kidney injury through increasing oxidative stress (Figure 3). Recent studies revealed that MTX underwent polyglutamation and transformed into polyglutamylated MTX (MTX-PGs) in the cell, and MTX-PGs showed severe cytotoxicity [23]. The MTX-PGs stayed at the cytoplasm for longer time until MTX-PGs changed back into MTX [23]. Because intracellular MTX-PGs concentration increases and sustained over time while administration [24], MTX in kidney cells may be more likely to transfer into MTX-PGs, while low dosage of MTX was administrated with longer duration. Therefore, long MTX 
administration duration may cause more severe kidney injury due to MTX intracellular polyglutamation. Our results also showed increased oxidative stress in MTX-accumulated kidney (Figure 4). The above finding suggests that higher intracellular MTX-PGs concentration may trigger higher oxidative stress and severe kidney injury. Future studies will focus on determining the involvement of MTX-PGs accumulation in kidney tissue and further exploring the molecular mechanism underlying Long-MTX administration-induced kidney injury.

Attempts have been made to establish animal models showing MTX-induced kidney injury, as of this moment, the suitable model to study the effect of administration duration on MTX-induced kidney injury is not yet available. In this study, we successfully established the animal model with renal failure caused by longer duration of MTX administration. We observed the significant decrease of body weight, water intake, and urine volume in this model (Figures 1a-1c). As expected, urea nitrogen and CRE in urine were obviously decreased in Long-MTX group (Figures $1 \mathrm{~d}$ and 1e), while BUN and CRE in serum were increased in Long-MTX group (Figures $1 \mathrm{f}$ and $1 \mathrm{~g}$ ). Additionally, in Long-MTX group, both N-gal and Kim-1, kidney injury markers, were also markedly increased (Figure 2). Previous studies have shown that $\mathrm{N}$-gal is one of the most reliable markers in kidney after ischemic or nephrotoxic acute kidney injury (AKI) [25], and N-gal expression is elevated in renal tissue after kidney injury [26]. In addition, it is welldocumented that Kim-1 is also a very useful kidney injury marker, and Kim-1 expression is rapidly increased after kidney injury [27]. Moreover, 4-HNE and MDA were significantly increased in LongMTX group (Figure 4), indicating that, at least in significant parts, this model showed the clinical phenotype of MTX-induced kidney injury. Interestingly, our model showed that both N-gal and Kim-1 were significantly increased in kidney tissue by longer duration of MTX administration (Figure 2). Recent studies have shown that both N-gal and Kim-1 are recognized as sensitive and early diagnostic markers for kidney injury $[25,26]$. Thus, N-gal and Kim-1 levels in urine or serum may have potential to be a useful biomarker for kidney injury caused by Long-MTX administration. In clinical case report, it has been reported that serum CRE is increased during MTX-induced kidney injury [13]. However, in this model, urine CRE was obviously decreased in Long-MTX group without the significant increase of serum CRE. It may due to the deceased CRE synthesis in Long-MTX group. Because tetrahydrofolate (THF) is necessary for CRE synthesis [28], and MTX shows its therapeutic effect by inhibiting the synthesis of THF [29], MTX administration may lead to a decrease in CRE synthesis. It should be noted that folic acid was used with MTX in clinical usage, but not in animal experiment $[9,13]$, indicating the difference between animal model and clinical case in CRE synthesis. Further investigation will be needed to determine the more detailed molecular mechanism and verify the usefulness of this model for studying MTX-induce kidney injury.

In conclusion, we provided first hand evidence that longer duration of MTX administration caused more severe kidney injury in rat model. MTX accumulation in renal tissue by Long-MTX administration caused kidney injury through an increase in oxidative stress. This finding may bring new insights into understanding of MTX-induced kidney injury, and may lead to clinical application for appropriate treatment with MTX.

\section{Acknowledgements}

This work was supported by Grant (JP25293040 and JP25670080) from the Japan society for the promotion of science KAKENHI. The first author was supported by State-Sponsored Scholarship Program (No.201306370035) from the China scholarship council.

\section{References}

1. Genestier L, Paillot R, Quemeneur L, Izeradjene K, Revillard JP (2000) Mechanisms of action of methotrexate. Immunopharmacology 47: 247-257.

2. Thyss A, Milano G, Kubar J, Namer M, Schneider M (1986) Clinical and pharmacokinetic evidence of a life-threatening interaction between methotrexate and ketoprofen. Lancet 1: 256-258.

3. Stovall TG, Ling FW, Gray LA, Carson SA, Buster JE (1991) Methotrexate treatment of unruptured ectopic pregnancy: a report of 100 cases. Obstet Gynecol 77: 749-753.

4. Sokka T (2010) Increases in use of methotrexate since the 1980s. Clin Exp Rheumatol 28: S13-20.

5. Albers HM, Wessels JA, van der Straaten RJ, Brinkman DM, SuijlekomSmit LW, et al. (2009) Time to treatment as an important factor for the response to methotrexate in juvenile idiopathic arthritis. Arthritis Rheum 61: 46-51.

6. Giannini EH (2002) Methotrexate: vital new information about a middle aged drug. J Rheumatol 29: 2031-2033.

7. Longo-Sorbello GS, Bertino JR (2001) Current understanding of methotrexate pharmacology and efficacy in acute leukemias. Use of newer antifolates in clinical trials. Haematologica 86: 121-127.

8. Asvadi I, Hajipour B, Asvadi A, Asl NA, Roshangar L, et al. (2011) Protective effect of pentoxyfilline in renal toxicity after methotrexate administration. Eur Rev Med Pharmacol Sci 15: 1003-1009.

9. Stark AN, Jackson G, Carey PJ, Arfeen S, Proctor SJ (1989) Severe renal toxicity due to intermediate-dose methotrexate. Cancer Chemother Pharmacol 24: 243-245.

10. Widemann BC, Adamson PC (2006) Understanding and managing methotrexate nephrotoxicity. Oncologist 11: 694-703.

11. Ethan den boer (2014) Therapeutic Drug-Monitoring of MethotrexatePolyglutamates in Rheumatoid Arthritis. Reumafonds, Erasmus University Rotterdam, Netherlands.

12. Perazella MA (1999) Crystal-induced acute renal failure. Am J Med 106: 459-465.

13. Strang A, Pullar T (2004) Methotrexate toxicity induced by acute renal failure. J R Soc Med 97: 536-537.

14. Albushra Y, Hasan Y (2013) Prevention and Management of High Dose Methotrexate Toxicity. J Cancer Sci Ther 5: 106-12.

15. Goldie JH, Price LA, Harrap KR (1972) Methotrexate toxicity: correlation with duration of administration, plasma levels, dose and excretion pattern. Eur J Cancer 8: 409-414.

16. Lin XB, Zhou NN, Li S, Cai QQ, Xia ZJ, et al. (2008) Effects of infusion duration of high-dose methotrexate on cerebrospinal fluid drug levels in lymphoma patients. Ai Zheng 27: 1100-1105.

17. Yarandi F, Mousavi A, Abbaslu F, Aminimoghaddam S, Nekuie S, et al. (2016) Five-Day Intravascular Methotrexate Versus Biweekly Actinomycin-D in the Treatment of Low-Risk Gestational Trophoblastic Neoplasia: A Clinical Randomized Trial. Int J Gynecol Cancer 26: 971-976.

18. Mikkelsen TS, Sparreboom A, Cheng C, Zhou Y, Boyett JM, et al. (2011) Shortening infusion time for high-dose methotrexate alters antileukemic effects: a randomized prospective clinical trial. J Clin Oncol 29: 1771-1778.

19. Singhal SS, Singh SP, Singhal P, Horne D, Singhal J, et al. (2015) Antioxidant role of glutathione S-transferases: 4-Hydroxynonenal, a key molecule in stress-mediated signaling. Toxicol Appl Pharmacol 289: 361-370.

20. Ayala A, Muñoz MF, Argüelles S (2014) Lipid peroxidation: production, metabolism, and signaling mechanisms of malondialdehyde and 4hydroxy-2-nonenal. Oxid Med Cell Longev 2014: 360438. 
Citation: Li X, Abe E, Yamakawa Y, Yoneda G, Fujino R, et al. (2016) Effect of Administration Duration of Low Dose Methotrexate on Development of Acute Kidney Injury in Rats. J Kidney 2: 130. doi:10.4172/2472-1220.1000130

Page 6 of 6

21. Buitenkamp TD, Math ̃'t RA, de Haas V, Pieters R, Zwaan CM (2010) Methotrexate-induced side effects are not due to differences in pharmacokinetics in children with Down syndrome and acute lymphoblastic leukemia. Haematologica 95: 1106-13.

22. Morsy MA, Ibrahim SA, Amin EF, Kamel MY, Rifaai RA, et al. (2013) Curcumin ameliorates methotrexate-induced nephrotoxicity in rats. Adv Pharmacol Sci 2013: 387071.

23. Gervasini G, Vagace JM (2012) Impact of genetic polymorphisms on chemotherapy toxicity in childhood acute lymphoblastic leukemia. Front Genet 3: 249.

24. Woolf RT, West SL, Arenas-Hernandez M, Hare N, Peters van Ton AM, et al. (2012) Methotrexate polyglutamates as a marker of patient compliance and clinical response in psoriasis: a single-centre prospective study. $\mathrm{Br} \mathrm{J}$ Dermatol 167: 165-173.

25. Mishra J, Ma Q, Prada A, Mitsnefes M, Zahedi K, et al. (2003) Identification of neutrophil gelatinase-associated lipocalin as a novel early urinary biomarker for ischemic renal injury. J Am Soc Nephrol 14: 2534-2543.

26. Han M, Li Y, Liu M, Li Y, Cong B (2012) Renal neutrophil gelatinase associated lipocalin expression in lipopolysaccharide-induced acute kidney injury in the rat. BMC Nephrol 13: 25.

27. Bonventre JV (2009) Kidney injury molecule-1 (KIM-1): a urinary biomarker and much more. Nephrol Dial Transplant 24: 3265-3268.

28. Stead LM, Au KP, Jacobs RL, Brosnan ME, Brosnan JT (2001) Methylation demand and homocysteine metabolism: effects of dietary provision of creatine and guanidinoacetate. Am J Physiol Endocrinol Metab 281: E1095-1100.

29. Sohn KJ, Croxford R, Yates Z, Lucock M, Kim YI (2004) Effect of the methylenetetrahydrofolate reductase C677T polymorphism on chemosensitivity of colon and breast cancer cells to 5-fluorouracil and methotrexate. J Natl Cancer Inst 96: 134-144. 